\title{
Efficacy of Acupuncture in Improving Symptoms and Quality of Life of Patients With Acne Vulgaris: a Randomized Sham Acupuncture-controlled Trial
}

\section{Ruimin Jiao}

Guang'anmen Hospital

\section{Xu Zhai}

China Academy of Chinese Medical Sciences

\section{Xuecheng Zhang}

Beijing University of Chinese Medicine Affiliated Dongzhimen Hospital

\section{Zhiyi Xiong}

Guang'anmen Hospital

Zhishun Liu ( $\nabla$ zhishunjournal@163.com )

Guang'anmen Hospital

\section{Research}

Keywords: Acupuncture, Acne vulgaris, Quality of life, Randomized controlled trial

Posted Date: January 6th, 2021

DOl: https://doi.org/10.21203/rs.3.rs-137376/v1

License: (c) (i) This work is licensed under a Creative Commons Attribution 4.0 International License. Read Full License

Version of Record: A version of this preprint was published at Acupuncture in Medicine on April 18th, 2022. See the published version at https://doi.org/10.1177/09645284221076506. 


\section{Abstract}

Objective: The aim of this study was to examine the effectiveness of acupuncture in treating the symptoms and QoL of patients with moderate or severe AV.

Methods: Participants were randomly assigned (1:1) to receive 12 treatment sessions of acupuncture or sham acupuncture over 4 weeks with 24 weeks of follow-up. The primary outcome was the change from baseline in the Skindex-16 scale total score at treatment completion. Secondary outcomes included Skindex-16 subscale score, Dermatology Life Quality Index scale total score, total lesion count and inflammatory lesion count, and visual analogue scale scores for itch and pain evaluation.

Results: There is no statistically significant between-group difference for the primary outcome and any secondary outcomes after 4 weeks of treatment and at 16 weeks and 28 weeks of follow-up, except for the Skindex-16 subscale (the emotions of participants with AV) at week $4(P=0.026)$. No serious adverse events occurred in two group.

Conclusion: Acupuncture and sham acupuncture might both relieve the symptoms of patients with moderate or severe $\mathrm{AV}$, reduce the number and degree of inflammatory lesion counts, and improve QoL.

Trial registration number: ChiCTR-1900023649 Chinese Clinical Trial Registry

\section{Introduction}

Acne vulgaris (AV) is a chronic inflammatory dermatosis notable for open or closed comedones and inflammatory lesions, including papules, pustules, or nodules. AV lesions predominate in exposed areas such as over the face, chest, and back ${ }^{1}$. An epidemiology study of AV identified a prevalence of acne vulgaris of $9.4 \%$ worldwide ${ }^{2}$. The recurrence of AV lesions on the face may induce feelings of guilt, shame, and social isolation. AV also involves other symptoms, such as, itching, pain, and other symptoms. These symptoms of AV may affect the physical and mental health of patients, adversely affecting their quality of life $(\mathrm{Q} o \mathrm{~L})^{3-7}$.

Conventional western medicine therapy for AV according to American Society of Dermatology guidelines include benzoyl peroxide, topical retinoids, or systemic antibiotics, which are recommend as the first-line treatment for mild-to-severe $\mathrm{AV}{ }^{1}$. However, these therapies may lead to skin drying, peeling, erythema, and other related side effects in patients with AV. In addition, long-term use of medicine may lead to drug resistance, which may result in a relatively high recurrence rate of $A V^{8}$. Therefore, natural and safe therapies of AV should be investigated.

A systematic review concluded that acupuncture could be used as a complementary alternative treatment due to its fewer adverse reactions compared with western medicine treatments ${ }^{9}$. In several clinical trials, acupuncture might reduce the skin lesions and improve the QoL of patients with $A{ }^{10}$. However, these trials were affected by potential bias due to small sample sizes, non-placebo/sham/waiting list control, or 
use of self-defined outcome measures. Thus, robust evidence of the efficacy of acupuncture in patients with $\mathrm{AV}$ is yet to be provided. Meanwhile, two systematic reviews recommended using the measure of QoL for evaluating the effect of acupuncture on AV ${ }^{11}{ }^{12}$, but so far, no clinical trials included the QoL measure to evaluate the effect of acupuncture of patients with AV. The aim of this study was to evaluate the effect of acupuncture compared with sham acupuncture on the symptoms and QoL of patients with moderate or severe AV.

\section{Methods}

\section{Study design}

This was a prospective, randomized, sham acupuncture-controlled trial with two parallel arms and a 1:1 allocation ratio. The trial was conducted and patients recruited at the Guang'anmen Hospital, China Academy of Chinese Medical Sciences. The trial protocol was approved by the Ethical Committee of the Guang'anmen Hospital (protocol approval No. 2018-137-KY-01) and was registered with the Chinese Clinical Trial Registry ChiCTR-1900023649) on January 2, 2019. The trial protocol has been described in detail elsewhere ${ }^{13}$. The study conformed to the Standard Protocol Items: Recommendations for Interventional Trials (SPIRIT) ${ }^{14}$ and the Standards for Reporting Interventions in Clinical Trials of Acupuncture (STRICTA) ${ }^{15}$. Participants were recruited by advertisements. Before enrollment, all participants provided signed informed consent and were preliminarily screened by research assistants and diagnosed by dermatologist.

\section{Participants}

Participants who met the diagnostic criteria for AV according to the American Society of Dermatology guidelines ${ }^{1}$ were included if they met all of the following criteria: participants were 18-48 years of age, and their Global Acne Grading System scores were between 19 and $38{ }^{16}$.

The participants were excluded if they met any of the following criteria: participants with a history of polymerization acne, explosive acne, drug acne, premenstrual acne, cosmetic acne, occupational acne, any other subtypes of acne or severe diseases that may affect acne, such as polycystic ovary syndrome, thyroid disease, or atypical congenital adrenal hyperplasia; participants with other skin diseases potentially influencing the assessment of $\mathrm{AV}$, such as rosacea, folliculitis, or other skin diseases; participants with severe heart, liver, kidney, hematopoietic system, or autoimmune disorders, or severe systemic malnutrition; participants who were pregnant, lactating women, or those planning to conceive within 12 months; participants using antibiotics, retinoic acid, steroids, or anti-inflammatory drugs in the preceding month, or who had received acupuncture treatment over the past three months.

\section{Randomization, allocation concealment, and blinding}

The randomization was prepared by the National Clinical Drug Testing Institute of the Guang'anmen Hospital using a computerized random number generator. Sealed opaque envelopes were used to ensure 
randomization concealment. The number of the randomization sequence and information on group allocation were sealed in ordered envelopes, and envelopes were opened according to the patient's order of entry into the trial. The envelopes were kept by a researcher not involved in the treatment or assessment. The participants, outcome assessors, and statisticians were blinded to the group allocation. However, acupuncturists were not blinded in this trial due to the characteristics of the acupuncture. Participants were asked to answer the following questions within 5 min after any treatment in week 4: "Do you think you have received traditional acupuncture?" The response options were "yes," "no," or "unclear" to assess the blinding effect of sham acupuncture.

\section{Interventions}

The regimen of acupuncture was based on the Guidelines for AV treatment in China (revised version 2014) ${ }^{17}$ and the consensus of experienced acupuncturists. The acupuncturists had completed an undergraduate education or higher in acupuncture and had at least 2-3 years' experience. Moreover, all researchers received one day of training before participant enrollment. Participants received 12 treatment sessions of acupuncture or sham acupuncture (30 minutes per session) three times per week for 4 weeks with 24 weeks of follow-up. Participants were encouraged not to receive any other therapies during the study period. However, rescue medications were available to all participants in case any deterioration of the AV condition became unbearable during the trial, and details of medication use (name, time, frequency and dosage) were recorded.

In the acupuncture group, participants received acupuncture at Dazhui (CV14), and the bilateral acupoints of Quchi (LI11), Hegu (LI4), Zusanli (ST36), and Neiting (ST44) with disposable acupuncture needles $(0.30 \times 40$ mm, Huatuo Brand; Suzhou Medical Appliance, Suzhou, Jiangsu, China). After routine disinfection, acupuncturists inserted the needle at a depth of 30-40 mm at an angle of 10-15 degrees into CV14; Acupuncturists inserted needles at a depth of 25-30 mm into the bilateral acupoints of LI11, LI4, ST36, and ST44. All needles were gently manipulated three times (once every 10 minutes) by slight lifting, thrusting, and twisting manipulations to produce a sensation of de-qi (characteristic needling sensation of sourness, numbness, swelling and heaviness) ${ }^{18}$.

In the sham acupuncture group, participants received minimal acupuncture at sham CV14 (10 mm away from CV14 ), LI11 (10 mm away from LI11), LI4 (10 mm away from LI4), ST36 (25 mm away from ST36), and ST44 (10 mm away form ST44). For sham CV14, LI11, LI4, ST36, and ST44 acupuncture, needles were inserted vertically at a depth of 1-2 $\mathrm{mm}$ without any manipulation and de-qi.

\section{Outcome measures}

The primary outcome was the change in total score of the Skindex-16 scale from baseline at the end of week 4. This scale includes a total of 16 items, which are categorized into three domains; the symptoms, the emotions, and the functions of participants with AV. The Skindex-16 scale (range: 0-100, lower scores representing better QoL) is a brief, skin-associated QoL scale used to evaluate the efficacy of acupuncture in improving the QoL of patients with $\mathrm{AV}^{19,20 .}$ 
The secondary outcomes were as follows:

1) The change from baseline in the Skindex-16 scale total score at weeks 16 and 28;

2) The change from baseline in the Skindex-16 sub scale (the symptoms, emotions, and function of participants with AV) scores at weeks 4, 16, and 28;

3) The change from baseline in the Dermatology Life Quality Index (DLQI, range: 0-30, higher scores representing better QoL) ${ }^{21}$ total score at weeks 16 and 28;

4) The change from baseline in the total lesion (inflammatory and non-inflammatory lesions) count (TLC) 22,23 at weeks 4,16 , and 28 ;

5) The change from baseline in the inflammatory lesion count (ILC) ${ }^{24}$ at weeks 4,16 , and 28 ;

6) The change from baseline in the degree of itching assessed using the Itch Assessment with Visual Analogue Scale (IVAS, range: 0-100, higher scores indicating higher itching intensity) ${ }^{23}$ at weeks 4, 16, and 28;

7) The change from baseline in the severity of pain assessed by a visual analog scale (PVAS) (range: 0100 , higher scores indicating greater pain) ${ }^{25}$ at weeks 4,16 , and 28 .

The participants' expectations regarding the acupuncture were evaluated using the following two questions: "Do you think acupuncture will be effective for treating the illness?" and "Do you think acupuncture will be effective for relieving the related symptoms of AV?" The response options were "yes", "no" or "unclear".

Adverse events (AEs) comprised AEs related to the acupuncture and those that were unrelated to the treatment; these were monitored and recorded throughout the trial.

\section{Statistical analysis}

We performed the sample size calculation using Power Analysis and Sample Size, version 11.0. The primary outcome was the change from baseline in the Skindex-16 scale score at the end of week 4 . The mean \pm standard deviation (SD) reductions in the Skindex-16 scale score after the 4-week treatment in the acupuncture and sham acupuncture groups were $12.50 \pm 19.09$ and $0.40 \pm 21.12$, respectively, according to our unpublished pilot trial. Assuming an alpha risk of $5 \%$ (two-sided test) and a beta risk of $20 \%$, a sample size of 100 (50 participants in each group) was calculated, considering a $20 \%$ drop-out rate.

Study data were analyzed according to the intention-to-treat principle. Baseline characteristics were assessed using an independent $t$ test or a non-parametric test for continuous variables and the chi-square test for categorical variables. For the between-group differences in the Skindex-16 scale total score, the Skindex-16 subscale (the symptoms, emotions, and function of participants with AV) score, DLQI score, 
TLC, ILC, IVAS score, and PVAS score data were analyzed using an independent $t$ test or non-parametric test, depending on data normality. The comparison with the baseline data was performed using a paired $t$ test or a non-parametric test, depending on data normality. Analysis of data pertaining to the participants' expectations of acupuncture and the blinding assessment was performed using the chi-square test or Fisher's exact test. Missing data were replaced with the actual observational data without imputation. Continuous variables are presented as the mean with SD or $95 \%$ confidence intervals $(\mathrm{Cl})$, while categorical variables are presented as numbers and proportions. Data analysis was performed using SPSS Statistics version 20.0 software (IBM Corp, Armonk, NY, USA). All probability ( $P$ )-values were twotailed, and a $P$-value $<0.05$ was considered to indicate statistical significance.

\section{Results}

In this study, 205 patients were screened between August 2019 and August 2020, of whom 105 patients were excluded and 100 patients remained in the trial. These 100 patients were randomized to either acupuncture or sham acupuncture, with 50 in each. Among the randomized participants, $20(20 \%)$ patients dropped out during study; nine (9\%) patients in the acupuncture group and 11 (11\%) patients in the sham acupuncture group. Twelve patients withdrew from the study due to lack of efficacy (six patients in the acupuncture group and six patients in the sham acupuncture group), and seven patients withdrew from the study due to coronavirus disease 2019 (COVID-19) precautions (two patients in the acupuncture group; five patients in the sham acupuncture group); for one patient (acupuncture group), no reason for drop out was provided (Fig. 1). No significant between-group differences were observed in the baseline characteristics of the 100 patients, as shown in Table 1 (each item, $P>0.05$ ). 
Table 1

Baseline patient characteristics

\section{Characteristics}

Age, mean $\pm S D$, y

Sex, n (\%)

Male

Female

Race, n (\%)

Han

Minorities

Marital status, n (\%)

Yes

No

Educational level, n (\%)

Primary education or below

Secondary education

Tertiary education

$\mathrm{BMI}$, mean $\pm \mathrm{SD}$

Time of sports activity, $\mathrm{n}(\%)$

0-3 times/month

\section{Acupuncture \\ $(n=50)$ \\ $P$ value acupuncture $(n=50)$}

$26.96 \pm 4.60$

$27.28 \pm 6.36$

0.774 


\begin{tabular}{|c|c|c|c|}
\hline Characteristics & $\begin{array}{l}\text { Acupuncture } \\
(n=50)\end{array}$ & $\begin{array}{l}\text { Sham } \\
\text { acupuncture } \\
(n=50)\end{array}$ & $\begin{array}{l}P \\
\text { value }\end{array}$ \\
\hline 1-2 times/week & $19(38)$ & $20(40)$ & \\
\hline 3-4 times/week & $6(12)$ & $4(8)$ & \\
\hline$\geq 5$ times/week & $3(6)$ & $2(4)$ & \\
\hline \multicolumn{4}{|l|}{ Daily sleep duration, n (\%) } \\
\hline$<7 \mathrm{~h}$ & $12(24)$ & $14(28)$ & 0.543 \\
\hline $7-9 h$ & $37(74)$ & $36(72)$ & \\
\hline$>9 \mathrm{~h}$ & $1(2)$ & $0(0)$ & \\
\hline \multicolumn{4}{|l|}{ Smoking history, n (\%) } \\
\hline Yes & $3(6)$ & $2(4)$ & 1 \\
\hline No & $47(94)$ & $48(96)$ & \\
\hline \multicolumn{4}{|l|}{ Drinking history, n (\%) } \\
\hline Yes & $2(4)$ & $4(8)$ & 0.678 \\
\hline No & $48(96)$ & $46(92)$ & \\
\hline AV duration, mean $\pm \mathrm{SD}$, mo & $\begin{array}{l}56.07 \pm \\
52.12\end{array}$ & $56.65 \pm 7.30$ & 0.956 \\
\hline $\begin{array}{l}\text { Severity of } A V \text { as assessed by Global Acne Grading System, } \\
\text { mean } \pm S D\end{array}$ & $24.66 \pm 4.94$ & $23.92 \pm 4.65$ & 0.443 \\
\hline \multicolumn{4}{|l|}{ Expectations of the effect of acupuncture in general, $\mathrm{n}(\%)$} \\
\hline Yes & $32(64)$ & $27(54)$ & 0.307 \\
\hline
\end{tabular}

Abbreviations: AV: acne vulgaris; BMI: body mass index; IVAS: Itch assessment with visual analogue scale; PVAS: Pain assessment with visual analogue scale; SD: standard deviation.

a Scores on the Skindex-16 range from 0 to 100, with higher scores indicating worse symptoms and quality of life, and this scale includes a total of 16 items that are categorized into three domains: the symptoms of participants with $\mathrm{AV}$, the emotions of participants with $\mathrm{AV}$, and function of participants with AV. Each the Skindex-16 sub-scale also range from 0 to 100, with higher scores indicating worse corresponding domains of symptoms, emotions and function.

b Scores on the Dermatology Life Quality Index scale range from 0 to 30 , with higher scores indicating worse quality of life.

c Scores on the IVAS range from 0 to 100 , with higher scores indicating worse itch intensity.

d Scores on the PVAS range from 0 to 100 , with higher scores indicating worse pain intensity. 


\begin{tabular}{|c|c|c|c|}
\hline Characteristics & $\begin{array}{l}\text { Acupuncture } \\
(n=50)\end{array}$ & $\begin{array}{l}\text { Sham } \\
\text { acupuncture } \\
(\mathrm{n}=50)\end{array}$ & $\begin{array}{l}P \\
\text { value }\end{array}$ \\
\hline No & $1(2)$ & $0(0)$ & \\
\hline Unclear & $17(34)$ & $23(46)$ & \\
\hline \multicolumn{4}{|l|}{ Expectations of acupuncture for $\mathrm{AV}, \mathrm{n}(\%)$} \\
\hline Yes & $31(62)$ & $28(56)$ & 0.685 \\
\hline No & $0(0)$ & 0 & \\
\hline Unclear & $19(38)$ & $22(44)$ & \\
\hline The total score of the Skindex-16 scale ${ }^{a}$, mean \pm SD & $\begin{array}{l}52.15 \pm \\
20.80\end{array}$ & $\begin{array}{l}49.84 \pm \\
20.49\end{array}$ & 0.576 \\
\hline $\begin{array}{l}\text { The total score of the Skindex- } 16 \text { sub-scale (the symptoms } \\
\text { of participants with AV) }{ }^{a} \text {, mean } \pm S D\end{array}$ & $\begin{array}{l}32.20 \pm \\
22.32\end{array}$ & $\begin{array}{l}30.90 \pm \\
19.42\end{array}$ & 0.931 \\
\hline $\begin{array}{l}\text { The total score of the Skindex-16 sub-scale (the emotions } \\
\text { of participants with } A V)^{a} \text {, mean } \pm S D\end{array}$ & $\begin{array}{l}69.98 \pm \\
23.51\end{array}$ & $\begin{array}{l}67.03 \pm \\
26.24\end{array}$ & 0.694 \\
\hline $\begin{array}{l}\text { The total score of the Skindex-16 sub-scale (the functioning } \\
\text { of participants with } A V \text { ) }{ }^{\text {a }} \text {, mean } \pm \text { SD }\end{array}$ & $\begin{array}{l}44.37 \pm \\
30.40\end{array}$ & $\begin{array}{l}41.63 \pm \\
31.76\end{array}$ & 0661 \\
\hline $\begin{array}{l}\text { The total score of Dermatology Life Quality Index scale }{ }^{b} \text {, } \\
\text { mean } \pm \text { SD }\end{array}$ & $9.78 \pm 4.26$ & $9.94 \pm 4.69$ & 0.801 \\
\hline Total lesion count, mean \pm SD & $\begin{array}{l}32.24 \pm \\
17.44\end{array}$ & $\begin{array}{l}33.88 \pm \\
15.93\end{array}$ & 0.407 \\
\hline Inflammatory lesion count, mean \pm SD & $13.46 \pm 7.71$ & $12.36 \pm 5.43$ & 0.675 \\
\hline The degree of itch assessed by IVAS ${ }^{c}$, mean \pm SD & $\begin{array}{l}27.32 \pm \\
24.02\end{array}$ & $\begin{array}{l}23.28 \pm \\
23.69\end{array}$ & 0.324 \\
\hline
\end{tabular}

Abbreviations: AV: acne vulgaris; BMI: body mass index; IVAS: Itch assessment with visual analogue scale; PVAS: Pain assessment with visual analogue scale; SD: standard deviation.

a Scores on the Skindex-16 range from 0 to 100, with higher scores indicating worse symptoms and quality of life, and this scale includes a total of 16 items that are categorized into three domains: the symptoms of participants with $\mathrm{AV}$, the emotions of participants with $\mathrm{AV}$, and function of participants with AV. Each the Skindex-16 sub-scale also range from 0 to 100, with higher scores indicating worse corresponding domains of symptoms, emotions and function.

b Scores on the Dermatology Life Quality Index scale range from 0 to 30 , with higher scores indicating worse quality of life.

${ }^{c}$ Scores on the IVAS range from 0 to 100 , with higher scores indicating worse itch intensity.

d Scores on the PVAS range from 0 to 100, with higher scores indicating worse pain intensity. 


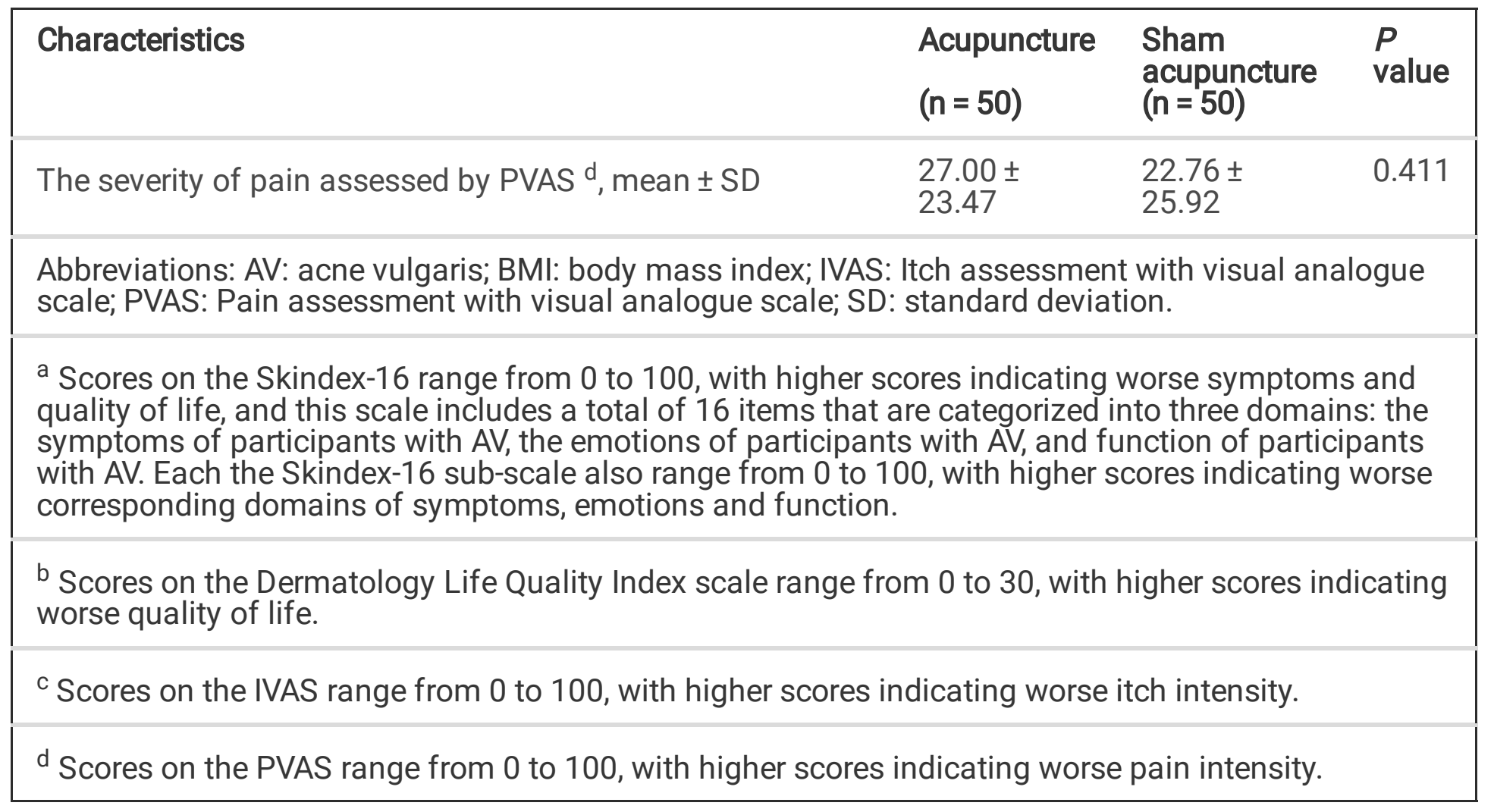

On average, participants in the acupuncture group and the sham acupuncture group received 11.2 and 10.8 treatment sessions, respectively, and a total of $92.0 \%$ of the participants in the acupuncture group and $88.0 \%$ in the sham acupuncture group received at least $10(>80 \%)$ of the planned treatment sessions.

\section{Primary outcome}

Differences in the decrease in the Skindex-16 scale scores from baseline to week 4 did not differ significantly between the acupuncture group $(n=41)$ and the sham acupuncture group $(n=39)(P>0.05)$. The change from baseline in the total score of the Skindex-16 scales after 4 weeks of treatment was $14.37(95 \% \mathrm{Cl},-20.58$ to -8.16$)$ in the acupuncture group and $-11.09(95 \% \mathrm{Cl},-18.76$ to -3.42$)$ in the sham acupuncture group, with a between-group difference of $-3.28(95 \% \mathrm{Cl},-12.93$ to 6.37$)$, as shown in Table 2. 
Table 2

Primary and Secondary Outcomes

Variable

Acupuncture

Sham

acupuncture

a

Difference

$(95 \% \mathrm{Cl})$

Palue

\section{Primary outcome}

The change from baseline in the Skindex-16

scale total score ${ }^{b}$

Week 4

$-14.37$

$(-20.58$ to

$-8.16)$

$-11.09$

$-3.28$

$(-18.76$ to

$-3.42)$

$(-12.93$ to

0.501

\section{Secondary outcomes}

The change from baseline in the Skindex-16

scale total score ${ }^{b}$

Week 16

$-9.61(-16.07$

to -3.15$)$

$-6.68(-12.54$

to -0.82$)$

6.37)

Week 16

Week 28

$\begin{array}{ll}-6.37(-12.03 & -2.86(-9.50 \\ \text { to } 0.71) & \text { to } 3.78)\end{array}$

-4.32
$(-11.55$ to
$5.68)$

0.870

$-3.51$

$(-12.06$ to

0.417

5.05)

The change from baseline in the Skindex-16

sub-scale (the symptoms of participants with

AV) score ${ }^{b}$

Week 4

$-4.13(-9.86$

to 1.32 )

$-1.29(-9.15$

to 6.57$)$

$-2.84$

$(-12.15$ to

0.568

6.46)

Week 16

$1.62(-6.05$ to
$9.30)$

$5.35(-3.48$

to 14.18$)$

$-3.73$

$(-15.21$ to

0.458

7.75)

Week 28

$5.80(0.92$ to

12.53)

$10.95(1.39$

to 20.51 )

$-5.15$

$(-16.56$ to

0.661

6.26)

The change from baseline in the Skindex-16

sub-scale (the emotions of participants with

AV) score ${ }^{b}$

Week 4

$-25.67$

$(-33.65$ to-

17.68)

$-15.42$

$(-24.77$ to

$-6.06)$

$-10.25$

$(-22.94$ to

0.026

$-19.94$

$(-28.62$ to

$-11.26)$

$-13.47$

$(-20.99$ to

$-5.95)$

$1.80)$

Week 16 


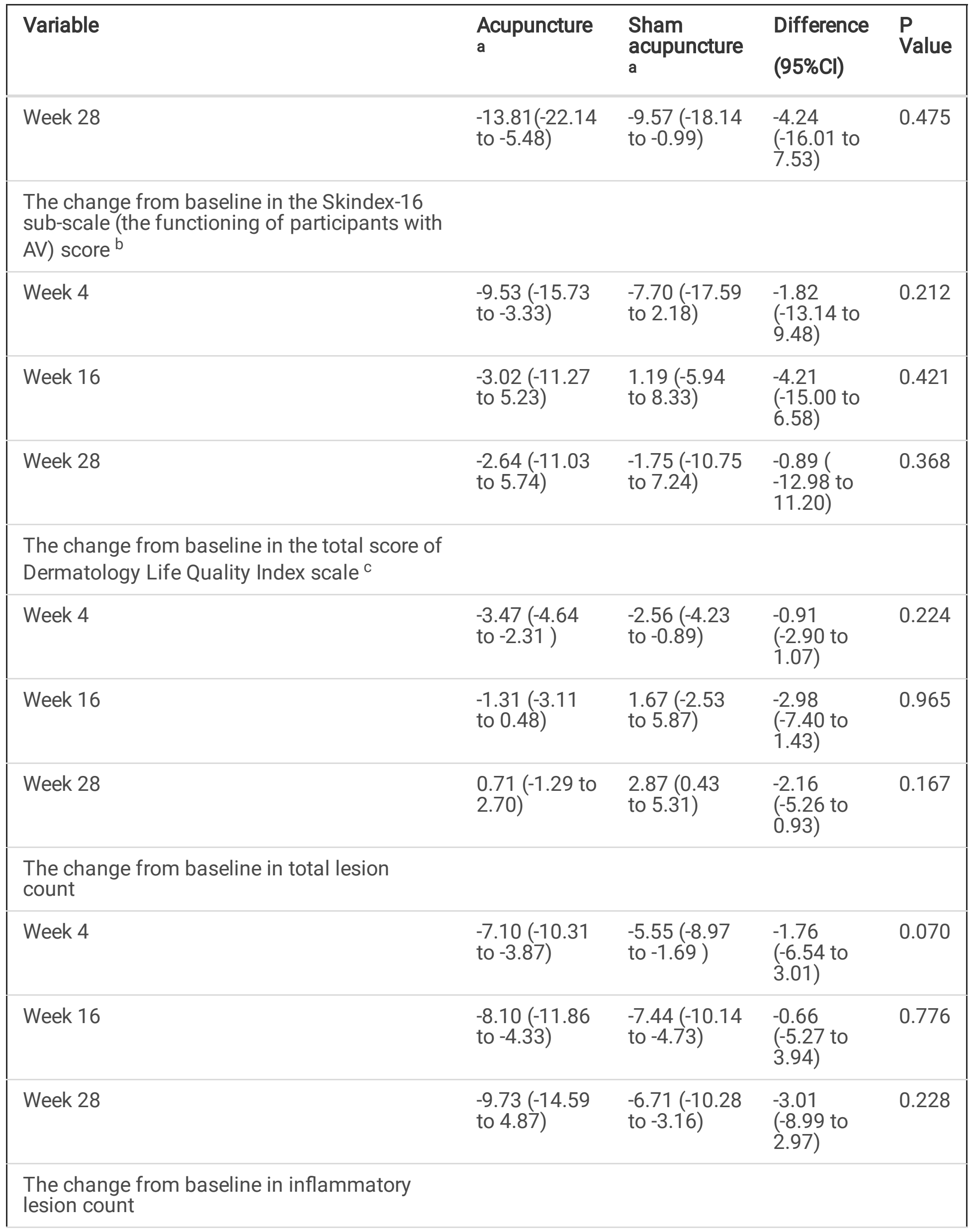




\begin{tabular}{|c|c|c|c|c|}
\hline Variable & $\begin{array}{l}\text { Acupuncture } \\
\text { a }\end{array}$ & $\begin{array}{l}\text { Sham } \\
\text { acupuncture } \\
\text { a }\end{array}$ & $\begin{array}{l}\text { Difference } \\
(95 \% \mathrm{Cl})\end{array}$ & $\begin{array}{l}\mathrm{P} \\
\text { Value }\end{array}$ \\
\hline Week 4 & $\begin{array}{l}-3.83(-6.02 \\
\text { to }-1.65)\end{array}$ & $\begin{array}{l}-1.33(-3.25 \\
\text { to } 0.58)\end{array}$ & $\begin{array}{l}-2.50 \\
(-5.38 \text { to } \\
0.38)\end{array}$ & 0.133 \\
\hline Week 16 & $\begin{array}{l}-2.92(-5.53 \\
\text { to }-0.32)\end{array}$ & $\begin{array}{l}-1.12(-3.22 \\
\text { to } 0.97)\end{array}$ & $\begin{array}{l}-1.79 \\
(-5.11 \text { to } \\
1.52)\end{array}$ & 0.636 \\
\hline Week 28 & $\begin{array}{l}-3.43(-5.62 \\
\text { to }-1.26)\end{array}$ & $\begin{array}{l}-2.13(-4.25 \\
\text { to }-0.01)\end{array}$ & $\begin{array}{l}-1.31 \\
(-4.30 \text { to } \\
1.68)\end{array}$ & 0.347 \\
\hline \multicolumn{5}{|c|}{$\begin{array}{l}\text { The change from baseline in degree of itch } \\
\text { assessed by IVAS }\end{array}$} \\
\hline Week 4 & $\begin{array}{l}-9.38(-15.40 \\
\text { to }-3.36)\end{array}$ & $\begin{array}{l}-6.77(-12.07 \\
\text { to }-1.47)\end{array}$ & $\begin{array}{l}-2.61 \\
(-10.56 \text { to } \\
5.33)\end{array}$ & 0.515 \\
\hline Week 16. & $\begin{array}{l}-10.71 \\
(-18.77 \text { to } \\
-2.64)\end{array}$ & $\begin{array}{l}-4.89(-13.71 \\
\text { to } 3.92)\end{array}$ & $\begin{array}{l}-5.80 \\
(-17.54 \text { to } \\
5.95)\end{array}$ & 0.328 \\
\hline Week 28 & $\begin{array}{l}-14.98 \\
(-21.91 \text { to } \\
-8.04)\end{array}$ & $\begin{array}{l}-3.82(-12.66 \\
\text { to } 5.02)\end{array}$ & $\begin{array}{l}-11.56 \\
(-22.15 \text { to } \\
0.16)\end{array}$ & 0.072 \\
\hline \multicolumn{5}{|c|}{$\begin{array}{l}\text { The change from baseline in severity of pain } \\
\text { assessed by PVAS }\end{array}$} \\
\hline Week 4 & $\begin{array}{l}-13.02(-19 \text { to } \\
-7.04)\end{array}$ & $\begin{array}{l}-12.79 \\
(-21.35 \text { to } \\
-4.24)\end{array}$ & $\begin{array}{l}0.23 \\
(-10.38 \text { to } \\
9.93)\end{array}$ & 0.964 \\
\hline Week 16 & $\begin{array}{l}-10.44 \\
(-17.71 \text { to } \\
-3.16)\end{array}$ & $\begin{array}{l}-8.51(-19.34 \\
\text { to } 2.32)\end{array}$ & $\begin{array}{l}-1.93 \\
(-14.65 \text { to } \\
10.80)\end{array}$ & 0.700 \\
\hline Week 28 & $\begin{array}{l}-12.66 \\
(-19.48 \text { to } \\
-5.84)\end{array}$ & $\begin{array}{l}-7.87(-17.98 \\
\text { to } 2.23)\end{array}$ & $\begin{array}{l}-4.78 \\
(-16.67 \text { to } \\
7.10)\end{array}$ & 0.547 \\
\hline \multicolumn{5}{|c|}{$\begin{array}{l}\text { Abbreviations: AV: acne vulgaris; IVAS: Itch assessment with visual analogue scale; PVAS: Pain } \\
\text { assessment with visual analogue scale. }\end{array}$} \\
\hline \multicolumn{5}{|c|}{$\begin{array}{l}\text { Analysis was performed following the intention-to-treat principle. Missing values were replaced with } \\
\text { the actual observational data without imputation. }\end{array}$} \\
\hline \multicolumn{5}{|c|}{$\begin{array}{l}\text { a Eight patients in the acupuncture group and eleven patients in the sham acupuncture group dropped } \\
\text { out at week 4; one patients in the acupuncture group was lost to follow-up at week 16; none of patient } \\
\text { in the acupuncture group and the sham acupuncture group was lost to follow-up at week 28; }\end{array}$} \\
\hline
\end{tabular}


Acupuncture

a
Sham

acupuncture

a
Difference

$(95 \% \mathrm{Cl})$
$P$ Value

b Scores on the Skindex-16 range from 0 to 100, with higher scores indicating worse symptoms and quality of life, and this scale includes a total of 16 items that are categorized into three domains: the symptoms of participants with $\mathrm{AV}$, the emotions of participants with $\mathrm{AV}$, and function of participants with AV. Each the Skindex-16 sub-scale also range from 0 to 100, with higher scores indicating worse corresponding domains of symptoms, emotions and function.

c Scores on the Dermatology Life Quality Index scale range from 0 to 30, with higher scores indicating worse quality of life.

${ }^{\mathrm{d}}$ Scores on the IVAS range from 0 to 100 , with higher scores indicating worse itch intensity.

e Scores on the PVAS range from 0 to 100 , with higher scores indicating worse pain intensity.

\section{Secondary outcomes}

For secondary outcomes, statistically significant differences were observed between the acupuncture and sham acupuncture groups in the change from baseline in the total score of the Skindex-16 subscale (the emotions of participants with AV) at week $4(P=0.026)$; however, no statistically significant differences between the acupuncture and sham acupuncture groups were observed in the change from baseline in the total score of the Skindex-16 subscale (the emotions of participants with AV) at week 16 or week 28 $(P>0.05)$. Meanwhile, no statistically significant differences were observed between the acupuncture and sham acupuncture groups in the change from baseline in the total score of the Skindex-16 subscale (symptoms and function of participants with AV) total score, the DLQI scale total score, the TLC, the ILC, the IVAS total score, or the PVAS score at all the measured time points (all, $P>0.05$ ), as shown in Table 2. No statistical significant difference was observed between the two groups in terms of the success of blinding ( $P=0.904$; Table 3$)$.

Table 3

Blinding assessment at week 4

\begin{tabular}{|llll|}
\hline $\mathbf{N}(\%)$ & Acupuncture $(\mathbf{n = 4 1 )}$ & Sham acupuncture $(\mathbf{n = 3 9 )}$ & $\mathbf{P}$ Value \\
\hline yes & $32 / 41(78.1 \%)$ & $30 / 39(76.9 \%)$ & \multirow{2}{*}{0.904} \\
\cline { 1 - 2 } no & $0 / 41(0 \%)$ & $0 / 41(0 \%)$ & \\
\cline { 1 - 2 } Unclear & $9 / 41(22.0 \%)$ & $9 / 39(23.1 \%)$ & \\
\hline
\end{tabular}

For the safety assessment, ten patients (six patients in the acupuncture group and four patients in the sham acupuncture group) reported AEs during the treatment. In the acupuncture group, three patients from the acupuncture group complained of needle pain after insertion, and three patients also had 
hematoma in the needle insertion area. In the sham acupuncture group, four patients complained of skin redness and itching after the intervention. No serious AEs occurred in either group, and none of the patients took rescue medication during the trial.

\section{Discussion}

This trial showed that both acupuncture and sham acupuncture might relieve the symptoms and improve the QoL of patients with moderate or severe AV, with no statistically significant differences between acupuncture and sham acupuncture. For the primary outcome, we choose the Skindex-16 scale as the primary outcome since it offered a sensitive and specific tool to assess the symptoms, characteristics (lesions), and QoL of patients with AV ${ }^{20}$. Although no statistically significant difference was observed between the acupuncture and sham acupuncture groups in terms of Skindex-16 assessment, both groups showed a significant statistical decline in the Skindex-16 score by the end of treatment and during the follow-up period. According to a previous pharmaceutical trial on dermatological disease 26,27 , the differences between active intervention and placebo in the change from baseline in the total score of Skindex-16 scale ranged from -3.2 to -7.9 , and the active intervention in the change from baseline in the total score of Skindex-16 scale ranged from -7.2 to -18.6 . Our study showed that the change from baseline in the total score of the Skindex-16 scale was $-14.37(95 \% \mathrm{Cl},-20.58$ to -8.16$)$ in the acupuncture group and $-11.09(95 \% \mathrm{Cl}-18.76$ to -3.42$)$ in the sham acupuncture group after the 4week treatment. Therefore, acupuncture and sham acupuncture might both result in relief of symptoms and improvement in QoL in patients with moderate or severe AV during treatment and at follow-up. However, no statistically significant difference between the two groups was observed, which might be due to the possibility that sham acupuncture may have more nonspecific biological and even biological effects than other types of placebo ${ }^{28,29}$.

For the secondary outcomes, our data revealed no significant statistical differences in the change from the baseline in the Skindex-16 subscale (the symptoms and function of participants with AV), DLQI scale, TLC, ILC, IVAS, and PVAS scores in weeks 4-28 between the acupuncture group and the sham acupuncture group, except the statistically significant between-group difference in the Skindex-16 subscale (the emotions of participants with AV) score in the first 4 weeks. According to a previous pharmaceutical trial on dermatological disease ${ }^{26}$, the differences in the change in Skindex-16 subscale (the emotions of participants with AV) scores between active intervention and placebo from baseline ranged from -8 to -18.5 , and the active intervention in the change from baseline in the total score of Skindex-16 scale ranged from -14.3 to -24.8 . Our study showed that the change from baseline in the total score of the Skindex-16 scale was $-25.67(95 \% \mathrm{Cl},-33.65$ to -17.68$)$ in the acupuncture group and - $15.42(95 \% \mathrm{Cl},-24.77$ to -6.06$)$ in the sham acupuncture group after the 4-week treatment. Therefore, at the end of treatment, the improvement effect on emotions in patients with AV of acupuncture may be better than that of sham acupuncture. Additionally, with regard to AV lesion count, the results of this study were consistent with a previous study reporting a significant decrease by the 12th acupuncture session ${ }^{10}$. As for pain or itching, the differences between acupuncture and sham acupuncture, in terms 
of their change from the baseline, for the IVAS and PVAS total scores showed an increasing trend, which was similar to the results of a previous trial ${ }^{30}$, which used 12 sessions of acupuncture combined with moving cupping and ear point tapping for patients with AV. The AEs of acupuncture and sham acupuncture in our study were mild and transient and did not require special treatment.

Our study has several limitations. First, this was a single-center study with a relatively small sample size, and patients were recruited only in the Beijing area. Hence, the results of the study are mostly based on specific populations; whether or not they can be applied to populations in other regions and nations remains unknown. Second, acupuncture is a complex intervention, the effect of placebo and expectation is part of the total effect of acupuncture 27,31 , so a waitlist (no treatment) control group may be more appropriate as a reference group for further studies; this type of control group would eliminate bias associated with possible spontaneous remission of $A V$ to better evaluate the effectiveness of acupuncture for patients with AV. Third, because of the characteristics of acupuncture, the acupuncturist were not blinded in our trial, a fact which may be a source of potential bias. Fourth, the effect of the relatively high drop-out rate due to the influence of COVID-19 may also bias the results.

\section{Conclusion}

Acupuncture and sham acupuncture might both relieve the symptoms of patients with moderate or severe $\mathrm{AV}$, reduce the number and degree of inflammatory lesion counts, and improve the QoL of $A V$ patients. Acupuncture is a safe treatment. Further studies with larger sample sizes and a waitlist (no treatment) control should be performed to evaluate the effects of acupuncture on AV.

\section{Abbreviations}

AV: Acne vulgaris; QoL: Quality of life; GAGS: Global Acne Grading System; SPIRIT: Standard Protocol Items: Recommendations for Interventional Trials; STRICTA: Standards for Reporting Interventions in Clinical Trials of Acupuncture; DLQI: Dermatology Life Quality Index scale; ILC: inflammatory lesion count; IVAS: Itch assessment with visual analogue scale; PVAS: Pain assessment with visual analogue scale; TLC: Total lesion count; SD: Standard deviation; Cl: Confidence interval; AE: Adverse event; COVID-19: Coronavirus disease 2019.

\section{Declarations}

\section{Availability of data and material:}

All relevant data will be shared for a period beginning 3 months after publication and ending 5 years after publication. The datasets used in the present study are available from the corresponding author on reasonable request.

\section{Ethics approval and consent to participate:}


The study protocol the protocol was approved by the Institutional Review Board of the Guang'anmen Hospital in China (approval no. 2018-137-KY-01) (see Additional file 1). All procedures followed were in accordance with the ethical standards of the responsible committee on human experimentation (institutional and national) and with the Helsinki Declaration. Informed consent was obtained from all patients for being included in the study.

\section{Consent for publication:}

Not applicable.

\section{Competing interests:}

The authors declare that they have no competing interests.

\section{Funding:}

This trial will be conducted without any external funding or internal funding, and all the researchers or participants will voluntarily participate in this trial.

\section{Authors' contributions:}

Zhishun Liu and Ruimin Jiao conceived the idea of this trial and the design this study. Xu Zhai are responsible for statistical analysis. Ruimin Jiao, Xuecheng Zhang and Zhiyi Xiong are responsible for the recruitment and treatment of participants. This manuscript was drafted by Ruimin Jiao and revised by Zhishun Liu. All authors have read and approved the final manuscript.

\section{Acknowledgments:}

Not applicable.

\section{References}

1. Zaenglein AL, Pathy AL, Schlosser BJ, Alikhan A, Baldwin HE, Berson DS, Bowe WP, Graber EM, Harper JC, Kang S, Keri JE, Leyden JJ, Reynolds RV, Silverberg NB, Stein Gold LF, Tollefson MM, Weiss JS, Dolan NC, Sagan AA, Stern M, Boyer KM, Bhushan R. Guidelines of care for the management of acne vulgaris. J Am Acad Dermatol 2016; 74(5): 945-973.e33

2. Alshammrie FF, Alshammari R, Khan FH. Epidemiology of Acne Vulgaris and Its Association With Lifestyle Among Adolescents and Young Adults in Hail, Kingdom of Saudi Arabia: A CommunityBased Study. Cureus 2020; 12(7)

3. Mwanthi M, Zaenglein AL. Update in the management of acne in adolescence. Curr Opin Pediatr 2018; 30(4): 492-498

4. Vilar GN, Santos LA, Sobral Filho JF. Quality of life, self-esteem and psychosocial factors in adolescents with acne vulgaris. An Bras Dermatol 2015; 90(5): 622-629 
5. Smithard A, Glazebrook C, Williams HC. Acne prevalence, knowledge about acne and psychological morbidity in mid-adolescence: a community-based study. Br J Dermatol 2001; 145(2): 274-279

6. Mallon E, Newton JN, Klassen A, Stewart-Brown SL, Ryan TJ, Finlay AY. The quality of life in acne: a comparison with general medical conditions using generic questionnaires. $\mathrm{Br} \mathrm{J}$ Dermatol 1999; 140(4): 672-676

7. Bez Y, Yesilova Y, Kaya MC, Sir A. High social phobia frequency and related disability in patients with acne vulgaris. Eur J Dermatol 2011; 21(5): 756-760

8. Lizelle F, Candice C, Marique A, Du PJ, Minja G. Treatment Modalities for Acne. Molecules 2016; 21(8): 1063

9. Mansu SSY, Liang H, Shefton P, Coyle ME, Wang K, Zhang AL, Guo X, Lu C, Xue CCL. Acupuncture for Acne Vulgaris: A Systematic Review and Meta-Analysis. Evidence-Based Complementray and Alternative Medicine,2018,(2018-3-12) 2018; 2018: 1-12

10. Son BK, Yun Y, Choi IH. Efficacy of ah shi point acupuncture on acne vulgaris. Acupunct Med 2010; 28(3): 126-129

11. Cao HJ, Yang GY, Wang YY, Liu JP. Acupoint Stimulation for Acne: A Systematic Review of Randomized Controlled Trials. Med Acupunct 2013; 25(3): 173-194

12. Cao H, Yang G, Wang Y, Jian PL, Liu Y. Complementary therapies for acne vulgaris. Cochrane Database Syst Rev 2015; 1(6): CD009436

13. Jiao R, Huang M, Zhang W, Liu Z. Efficacy of acupuncture in improving the symptoms and the quality of life of patients with moderate or severe acne vulgaris: study protocol for a randomized controlled trial. Trials 2020; 21(1): 563

14. Chan AW, Tetzlaff JM, Gøtzsche PC, Altman DG, Mann H, Berlin JA, Dickersin K, Hróbjartsson A, Schulz KF, Parulekar WR, Krleza-Jeric K, Laupacis A, Moher D. SPIRIT 2013 explanation and elaboration: guidance for protocols of clinical trials. BMJ 2013; 346: e7586

15. Macpherson H, Altman DG, Hammerschlag R, Li Y, Wu T, White A, Moher D. Revised STandards for Reporting Interventions in Clinical Trials of Acupuncture (STRICTA): Extending the CONSORT Statement. PLoS Med 2010; 7(4): 35-46

16. Amol, Doshi, Ahmed, Zaheer, Matthew, J., Stiller. A comparison of current acne grading systems and proposal of a novel system. Int J Dermatol 1997;

17. Xiang L. Guideline for diagnosis and treatment of acne (the 2014 revised editon). J Clin Dermatol 2015; (1): 52-57

18. Zhou K, Fang J, Wang X, Wang Y, Zhu B. Characterization of de qi with electroacupuncture at acupoints with different properties. Journal of Alternative \& Complementary Medicine 2011; 17(11): 1007

19. Chren MM, Lasek RJ, Quinn LM, Mostow EN, Zyzanski SJ. Skindex, a quality-of-life measure for patients with skin disease: reliability, validity, and responsiveness. J Invest Dermatol 1996; 107(5): 707-713 
20. Chren MM, Lasek RJ, Sahay AP, Sands LP. Measurement properties of skindex-16: A brief quality-oflife measure for patients with skin diseases. Journal of Cutaneous Medicine \& Surgery 2001; 5(2): $105-110$

21. Finlay AY, Khan GK. Dermatology Life Quality Index (DLQI)-a simple practical measure for routine clinical use. Clin Exp Dermatol 1994; 19(3): 210-216

22. Palli MB, Reyes-Habito CM, Lima XT, Kimball AB. A single-center, randomized double-blind, parallelgroup study to examine the safety and efficacy of $3 \mathrm{mg}$ drospirenone $/ 0.02 \mathrm{mg}$ ethinyl estradiol compared with placebo in the treatment of moderate truncal acne vulgaris. J Drugs Dermatol 2013; 12(6): 633-637

23. Reich A, Heisig M, Phan NQ, Taneda K, Takamori K, Takeuchi S, Furue M, Blome C, Augustin M, Ständer S, Szepietowski JC. Visual analogue scale: evaluation of the instrument for the assessment of pruritus. Acta Derm Venereol 2012; 92(5): 497-501

24. Ramli R, Malik AS, Hani AF, Jamil A. Acne analysis, grading and computational assessment methods: an overview. Skin Res Technol 2012; 18(1): 1-14

25. Inhibitory effects of Cheongsangbangpoong-tang on both inflammatory acne lesions and facial heat in patients with acne vulgaris: A randomized controlled trial protocol. Bmc Complementary \& Alternative Medicine 2015; 16(1): 21

26. Kim YS, Ji JH, Oh SY, Lee S, Lee SI. A Randomized Controlled Trial of Epidermal Growth Factor Ointment for Treating Epidermal Growth Factor Receptor Inhibitor-Induced Skin Toxicities. Ssrn Electronic Journal 2018

27. Rollmann DC, Novotny PJ, Petersen IA, Garces YI, Bauer HJ, Yan ES, Wahner-Roedler D, Vincent A, Sloan JA, Issa Laack NN. Double-Blind, Placebo-Controlled Pilot Study of Processed Ultra Emu Oil Versus Placebo in the Prevention of Radiation Dermatitis. Int J Radiat Oncol Biol Phys 2015; 92(3):650-8

28. Linde K, Niemann K, Schneider A, Meissner K. How large are the nonspecific effects of acupuncture? A meta-analysis of randomized controlled trials. BMC Med 2010; 8(1): 75

29. 29. Zhang CS, Tan HY, Zhang GS, Zhang AL, Xue CC, Xie YM. Placebo Devices as Effective Control Methods in Acupuncture Clinical Trials: A Systematic Review. PLoS One 2015; 10(11): e0140825

30. Xie C. Efficacy of different frequencies acupuncture combined with moving cupping and ear point tapping in patients with moderate or severe acne vulgaris (in Chinese). Beijing University of Chinese Medicine; 2018.

31. Vickers, A. J. Placebo Controls in Randomized Trials of Acupuncture. Eval Health Prof 2002; 25(4): 421-435.

\section{Figures}




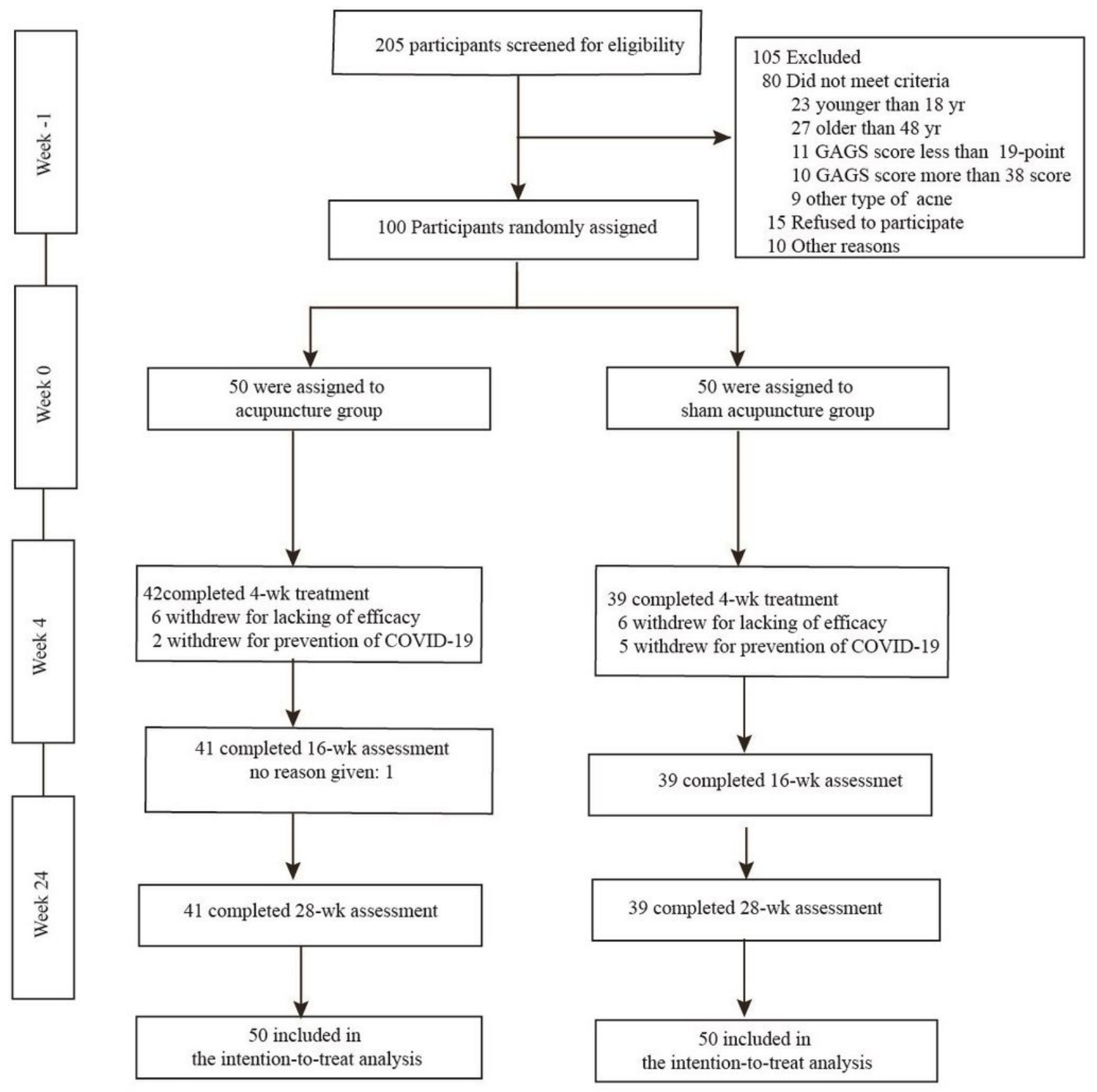

Figure 1

Flow of participants through the trial 